\title{
THE GREATEST AND THE LEAST VARIATE UNDER GENERAL LAWS OF ERROR*
}

\author{
BY
}

\author{
EDWARD LEWIS DODD
}

\section{INTRODUCTION}

To fit frequency distributions, several functions or curves have been used, most of which are generalizations of the so-called normal or Gaussian or Laplacean probability function

$$
\frac{h}{\sqrt{\pi}} e^{-h^{2} x^{*}}=\frac{1}{\sigma \sqrt{2 \pi}} e^{-x^{2} / 2 \sigma^{2}}
$$

The differential equation satisfied by this function was generalized by Karl Pearson.t Gram, $\ddagger$ Charlier, $\S$ and Bruns $\|$ used the normal function and its successive derivatives, with constant coefficients, to form a series, of which, in practice, only a few terms are used. Jorgensen $\uparrow$ developed a logarithmic transformation, in which $x$ is replaced by $\log x$. Associated with the Law of Small Numbers is the Poisson exponential function $e^{-\lambda} \lambda x / x$ ! for which Bortkiewicz** gave a four-place table, and Sopertt a six-place table. The Charlierłł

\footnotetext{
* Presented to the Society, April 28, 1923. The word variate will refer to any of the particular values which a variable may take on; e. g., the height of some specified soldier in a regiment, - the greatest variate here would be the height of the tallest soldier in the regiment.

† Contributions to the mathematical theory of evolution, II: Skew variation in homogeneous material, Philosophical Transactions A, vol. 186 (1895), part I, pp. 343-144.

$\ddagger$ Uber die Entwickelung reeller Funktionen in Reihen mittelst der Methode der kleinsten Quadrate, Journal für die reine und angewandte Mathematik, vol.91, pp.41-73.

$\S$ über die Darstellung willkïrlicher Funktionen, Arkiv för Matematik, Astronomi och Fysik, vol. 2, number 20.

II Uber die Darstellung von Fehlergesetzen, Astronomische Nachrichten, vol. 143. IT See Arne Fisher, The Mathematical Theory of Probabilities, I (2d edition), pp. 236-260.

** Das Gesetz der Kleinen Zahlen, 1898. See Arne Fisher, loc. cit., p. 266.

† Pearson's Tables for Statisticians and Biometricians, pp. 113-121.

\# Meddelanden från Lunds Observatorium, 1905. Vorlesungen über die Grundzüge der Mathematischen Statistik, p. 6, 79-85. See also Arkiv, loc. cit.
} 
$B$-Series, for integral variates, makes use of the Poisson function and its differences. The Makeham* life function is well known in life insurance.

Dealing only with the normal function itself, Bortkiewiczt determined mean and modal values for the interval of variation, i. e., the difference between the greatest and the least of $n$ variates. For this same problem there remains to be considered the median and the asymptotic value of the interval of variation. The asymptotic value is a function of $n$ which, with a probability converging to certainty, gives the interval of variation with a relative error small at pleasure. To make the problem broader, we shall consider the greatest and the least variate individually, and shall set up six general classes of functions which include as special cases the frequency functions in common use.

These six classes of functions are distinguished as follows. Apart from a factor $\psi(x)$ satisfying certain inequalities, the probability-function for large values of $x$ is, respectively,
(1) 0 ;
(2) $x^{-1-a}$;
(3) $g^{x^{\alpha}}$;
(4) $g^{\log _{e} x \gamma^{\gamma}}$;
(5) $g^{c^{3}}$
(6) $x^{-x}$;

with $\alpha>0, \gamma>1,0<g<1, c>1$. The first represents a finite interval; the second is involved in Pearson types; the third, with $\alpha=2$, is the normal probability function; the fourth leads to logarithmically transformed functions; the fifth, to the Makeham life function; the sixth to the Poisson exponential function.

\section{DEFINITIONS}

Definition 1: Probability function. The function $\varphi(x)$ is a probability function for specified variates, if for each variate

$$
\Phi(x)=\int_{x}^{\infty} \varphi(t) d t
$$

is the probability that the variate will take on a value equal to or greater than $x$.

Even when the statistical material must be given in integers, it is customary to think of $\varphi(x)$ and $\Phi(x)$ as continuous, especially when the number of variates is large. When it is desirable to provide at the same time for

* Journal of the Institute of Actuaries, 1860. See Institute of Actuaries' Text Book, Part II, Chap. VI.

† Variationsbreite und mittlerer Fehler, Sitzungsberichte der Berliner Mathematischen Gesellschaft, Jahrgang 21, Sitzung am 26. Oktober 1921. 
continuous and discontinuous probability, the Stieltjes integral* may be used.

Definition 2: Asymptotic certainty. An "event" dependent upon $n$ variables or variates is asymptotically certain if for any positive $\eta$, small at pleasure, it is possible to determine an $n^{\prime}$ so that when $n>n^{\prime}$, the probability that the event will happen is greater than $1-\eta$.

\section{ThE ASYMPTOTIC VALUE OF THE GREATEST VARIATE}

THEOREM I. If the probability function $\varphi(x) \equiv 0$, for $x>x_{2}$, and if $\int_{x}^{x_{2}} \varphi(x) d x \neq 0$ when $x<x_{2}$, then it is asymptotically certain that the greatest of $n$ variates will differ from $x_{2}$ by less than any preassigned positive $\varepsilon$.

Proof. By hypothesis,

$$
\int_{x_{2}-\varepsilon}^{x_{2}} \varphi(x) d x=\delta \neq 0 \text {. }
$$

Then the probability that all the $n$ variates will be less than $x_{2}-\varepsilon$ is $(1-\delta)^{n}$, which approaches zero with increasing $n$.

A similar statement can be made for the least variate. In fact, in all the theorems which follow the treatment of the least variate will be omitted as obvious. Of course, $x$ will often be replaced here by $|x|$.

THEOREM II. If, for positive $x$, the probability function ist

$$
\varphi(x)=x^{-1-a} \cdot \psi(x),
$$

with $\alpha, k_{1}, k_{2}$, positive constants, and $k_{1}<\psi(x)<k_{2}$, then it is asymptotically certain that the greatest of $n$ variates will be

$$
n^{\left(1+\varepsilon^{\prime}\right) / a}
$$

where $\left|\varepsilon^{\prime}\right|<\varepsilon$, small at pleasure.

\footnotetext{
* See R. von Mises, Fundamentalsätze der Wahrscheinlichkeitsrechnung, Mathematische Zeitschrift, vol. 4 (1919), pp. 1-97.

$\dagger$ Here, and in the following theorems, if $\varphi(x)$ has the indicated form merely when $x$ is greater than some given constant, the theorem remains valid.
} 
Proof. By hypothesis,

$$
\int_{x}^{\infty} \varphi(x) d x<k_{2} \int_{x}^{\infty} x^{-1-\alpha} d x=\frac{k_{2}}{\alpha} x^{-a} .
$$

Hence, if with $\varepsilon>0$ small at pleasure, we set

$$
x=n^{(1+\varepsilon) / \alpha}
$$

it follows that

$$
\int_{x}^{\infty} \varphi(x) d x<\frac{k_{g}}{\alpha n^{1+\varepsilon}}
$$

Thus, the probability that a specified variate will be less than this $x$ is greater than

$$
1-\frac{k_{2}}{\alpha n^{1+8}}
$$

And the probability that all variates will be less than this $x$ is greater than

$$
\left(1-\frac{k_{8}}{\alpha n^{2+e}}\right)^{n}
$$

But this approaches unity as $n$ approaches infinity. And thus, with $\eta>0$ small at pleasure, it is possible to find $n^{\prime}$ so that if $n>n^{\prime}$, the probability that all variates will be less than $n^{(1+\varepsilon) / \alpha}$ is greater than $1-\frac{1}{2} \eta$.

Similarly, using

$$
\lim _{n \rightarrow \infty}\left(1-\frac{k_{1}}{\alpha n^{1-\varepsilon}}\right)^{n}=0
$$

it can be shown that the probability that all variates will be less than

$$
n^{(1-\varepsilon) / a}
$$

is less than $\frac{1}{2} \eta$ for $n$ greater than some $n^{\prime \prime}$. 
Thus, for large enough $n$, the greatest variate will lie in the interval from $n^{(1-\varepsilon) / \alpha}$ to $n^{(1+\varepsilon) / \alpha}$, unless all variates are less than $n^{(1-\varepsilon) / \alpha},-$ for which the probability is less than $\frac{1}{2} \eta,-$ or unless some variate surpasses $n^{(1+\varepsilon) / a},-$ for which the probability is likewise less than $\frac{1}{2} \eta$. Hence, by Definition 2 , it is asymptotically certain that the greatest variate will lie in the interval from $n^{(1-\varepsilon) / a}$ to $n^{(1+\varepsilon) / a}$.

THEOREM III. If the probability function is

$$
\varphi(x)=g^{x^{\alpha}} \cdot \psi(x), \text { with } x^{-\beta}<\psi(x)<x^{\beta},
$$

where $\alpha, \beta, g$ are positive constants, and $g<1$, then it is asymptotically certain that the greatest of $n$ variates will equal.

$$
\left(-\log _{\theta} n\right)^{1 / a}\left(1+\varepsilon^{\prime}\right), \text { with }\left|\varepsilon^{\prime}\right|<\varepsilon,
$$

small at pleasure.

Proof. Let

$$
I=\int_{x}^{\infty} g^{t^{\alpha}} t^{\beta} d t, \quad v=g^{t^{\alpha}}, \quad u=\frac{t^{\beta-\alpha+1}}{\alpha \log _{e} g} .
$$

Then, integrating by parts,

$I=C g^{x^{\alpha}} x^{\beta-a+1}+C(\beta-\alpha+1) \int_{x}^{\infty} g^{t^{\alpha}} t^{\beta-\alpha} d t ; \quad C=\frac{1}{\alpha\left(-\log _{e} g\right)}>0$.

Thus,

$$
I<C g^{x^{\alpha}} x^{\beta-a+1}
$$

provided $\beta-\alpha+1<0$. But, even if $\beta-\alpha+1>0$, the process performed $k$ times will put into the numerator $\beta-k \alpha+1$, which is ultimately negative. Hence

$$
I<g^{x^{\alpha}} \cdot F(x),
$$

where $F(x)$ is a sum of powers of $x$ with constant coefficients. 
Suppose, now, that

$$
x=\left(-\log _{g} n\right)^{1 / a}(1+\varepsilon),
$$

$\varepsilon>0$, small at pleasure. Then,

$$
g^{x^{\alpha}}=\frac{1}{n^{1+2 e_{1}}}
$$

where $1+2 \varepsilon_{1}=(1+\varepsilon)^{\alpha}, \varepsilon_{1}>0$. But, for large enough $n, F(x)<n^{\varepsilon_{1}}$. Then

$$
I<\frac{1}{n^{1+\varepsilon_{1}}} .
$$

Hence, the probability that the $n$ variates will all be less than $x$ is greater than

$$
\left(1-\frac{1}{n^{1+\varepsilon_{1}}}\right)^{n}>1-\frac{1}{2} \eta
$$

for $n>$ some $n^{\prime}$.

Now, let $J$ be the result of replacing $\beta$ by $-\beta$ in $I$. If, after integrating by parts, $-\beta-\alpha+1>0$, then

$$
J>C g^{x^{\alpha}} x^{-\beta-a+1} .
$$

But if $-\beta-\alpha+1<0$, a second integration by parts yields $-\beta-2 \alpha+1$, which is again negative. By combining the two results,

$$
J>G(x) \cdot g^{x^{\alpha}},
$$

where $G(x)$ is a sum of powers of $x$ with constant coefficients. If, now,

$$
x=\left(-\log _{g} n\right)^{1 / a}(1-\varepsilon),
$$

then for large $n$,

$$
J>1-\frac{G(x)}{n^{1-2 \varepsilon_{2}}}>1-\frac{1}{n^{1-\varepsilon_{2}}},
$$


where $1-2 \varepsilon_{2}=(1-\varepsilon)^{\alpha}, \varepsilon_{8}>0$. Thus, the probability that the $n$ variates will all be less than $x$ is less than

$$
\left(1-\frac{1}{n^{1-\varepsilon_{2}}}\right)^{n}<\frac{1}{2} \eta
$$

when $n>$ some $n^{\prime \prime}$.

THEOREM IV. If the probability function is

$$
\varphi(x)=g^{\left(\log _{\mathrm{o}} x\right)^{\gamma}} \cdot \psi(x)
$$

with $x^{-\beta}<\psi(x)<x^{\beta}$, where $c, g, \beta, \gamma$ are positive constants, $0<g<1, c>1$, $\gamma>1$, then it is asymptotically certain that the greatest of $n$ variates will equal

$$
c^{\left(-\log _{g} n\right)^{\left(1+\varepsilon^{\prime}\right) / \gamma}}
$$

with $\left|\varepsilon^{\prime}\right|<\varepsilon$, small at pleasure.

Proof. The proof follows the same general course as in the preceding theorem, with

$$
v=g^{\left(\log _{0} t\right)^{\gamma}}, \quad I=\int_{x}^{\infty} v t^{\beta} d t, \quad J=\int_{x}^{\infty} v t^{-\beta} d t .
$$

Upon integrating $I$ by parts, the new integral contains the same integrand multiplied by a factor which is increased if the negative portion is dropped, and $t$ is replaced by $x$. Thus

$$
I<\xi(x)+\zeta(x) \cdot I
$$

where, indeed, $\zeta(x)<1$ when $x$ is large and where the principal factors of $\xi(x)$ are $g^{\left(\log _{e} x\right)^{\gamma}}$ and powers of $x$. As for $J$, we may first take $\beta>1$, and again in the new factor set $t=x$.

Now, setting

$$
g^{\left(\log _{0} x\right)^{\gamma}}=\frac{1}{n^{m}}, \quad g^{\left(\log _{0} x\right)^{\gamma}} \cdot x^{\tau}=\frac{1}{n^{m^{\prime}}}
$$

it follows that

$$
\left(\log _{c} x\right)^{\gamma}=-m \log _{g} n, \quad\left(\log _{c} x\right)^{\gamma}+\tau \log _{g} x=-m^{\prime} \log _{g} n .
$$


By division,

$$
1+\frac{\tau \log _{c} x \cdot \log _{g} c}{\left(\log _{c} x\right)^{\gamma}}=\frac{m^{\prime}}{m}
$$

Hence, since $\gamma>1$,

$$
\lim _{x \rightarrow \infty} \frac{m^{\prime}}{m}=1
$$

Thus, asymptotically, the effect of $x^{\tau}$ disappears when combined with $g^{\left(\log _{e} x\right)^{\gamma}}$. Now set

$$
x=c^{\left(-\log _{g} n\right)^{(1+\varepsilon) / \gamma}} .
$$

Then

$$
\left(\log _{c} x\right)^{\gamma}=\left(-\log _{g} n\right)^{1+\varepsilon}>(1+\varepsilon)\left(-\log _{g} n\right)
$$

provided $n$ is large enough. Thus, since $g<1$,

$$
g^{\left(\log _{e} x\right)^{\gamma}}<\frac{1}{n^{1+\varepsilon}}
$$

With $\varepsilon_{1}$ suitably chosen, positive and less than $\varepsilon$, the probability that all variates will be less than $x$ is greater than

$$
\left(1-\frac{1}{n^{1+\varepsilon_{1}}}\right)^{n}
$$

which approaches unity as a limit.

THEOREM V. If the probability function is

$$
\varphi(x)=g^{c^{x}} \cdot \psi(x), \text { with } b^{-x}<\psi(x)<b^{x},
$$

where $b, c$, and $g$ are constants, $0<g<1, b>1, c>1$, then it is asymptotically certain that the greatest of $n$ variates will equal

$$
\left[\log _{c}\left(-\log _{g} n\right)\right]\left(1+\varepsilon^{\prime}\right), \text { with }\left|\varepsilon^{\prime}\right|<\varepsilon,
$$

small at pleasure. 
Proof. The proof follows the same general course as in Theorem III, with

$$
v=g^{c^{t}}, \quad I=\int_{x}^{\infty} v b^{t} d t, \quad J=\int_{x}^{\infty} v b^{-t} d t, \quad b_{1}=\frac{b}{c} .
$$

Then, upon integrating by parts, we find

$$
I<\frac{g^{c^{x}} b_{1}^{x}}{(-\log g)(\log c)} \text { if } \frac{b}{c}<1 .
$$

At all events, $\frac{b}{c^{k}}<1$, for large enough $k$, so that by repetitions of the process,

$$
I<x^{x} \cdot F(x),
$$

where $F(x)$ is a sum of terms such as $b_{1}^{x}$ with constant coefficients. Likewise $J$ can be proved greater than a similar expression, noting that $\log (b c)>0$. By first setting $x=\left[\log _{c}\left(-\log _{g} n\right)\right](1+\varepsilon)$, and then $x=\left[\log _{c}\left(-\log _{g} n\right)\right](1-\varepsilon)$ and noting that for large $n,\left(-\log _{g} n\right)<n^{\delta}, \delta>0$, small at pleasure, the required inequalities can be obtained.

THEOREM VI. If the probability function is

$$
\varphi(x)=x^{-x} \cdot \psi(x), \text { with } b^{-x}<\psi(x)<b^{x}, \text { constant } b>1,
$$

then it is asymptotically certain that the greatest of $n$ variates will be

$$
X\left(1+\varepsilon^{\prime}\right), \text { where } X^{X}=n, \text { with }\left|\varepsilon^{\prime}\right|<\varepsilon,
$$

small at pleasure.

Proof. Set

$$
v=t^{-t}, \quad I=\int_{x}^{\infty} v b^{t} d t, \quad J=\int_{x}^{\infty} v b^{-t} d t .
$$

Integrating by parts, dropping a negative term, replacing $t$ by $x$, we obtain

$$
I<z x^{-x} b^{x}+I z \log b, \text { where } z=\frac{1}{1+\log x} .
$$


Likewise,

$$
J>z x^{-x} b^{-x}-\left\{z \log b+z^{2} x^{-1}\right\} J .
$$

Moreover, if $x^{x}=n^{y}$ and $x^{x} b^{x}=n^{y^{\prime}}$, then asymptotically $\frac{d x}{x}=\frac{d y}{y}, y^{\prime}=y$. That is, a percentage error in $y$ is controlled by an equal percentage error in $x$; and $b^{x}$, when combined with $x^{x}$, makes no asymptotic contribution to the exponent of $n$.

\section{The MEDIAN* VALUE OF THE GREATEST VARIATE}

The probability that every one of $n$ variates will be less than $G$ is, as is well known,

$$
\left(1-\int_{G}^{\infty} \varphi(t) d t\right)^{n},
$$

where $\varphi(x)$ is the probability function. If, now, we determine $G$ so that this expression is equal to $\frac{1}{2}$, then it is equally likely that the greatest variate will or will not exceed $G$. This median value of the greatest variate is thus obtained by finding $G$ so that

$$
\int_{G}^{\infty} \varphi(t) d t=1-2^{-1 / n} .
$$

While a median, mean, or modal value for the greatest variate may be more difficult to compute than the asymptotic value, in the foregoing theorems, the former will, in general have more significance in practical problems. However, even here, the asymptotic value may be useful for a rough simple check.

\section{THE NORMALt PROBABILITY FUNCTION}

If, in Theorem III, we set

$$
h^{2}=\frac{1}{2 \sigma^{2}}=-\log _{e} g, \quad \alpha=2,
$$

\footnotetext{
*In the theory of errors, the so-called "probable error" is the median of the absolute values of the errors. Thus, it is equally likely that an error, taken positively, will or will not exceed the probable error.

† Rietz, in his article Frequency distributions obtained by certain transformations of normally distributed variates, Annals of Mathematics, ser. 2, vol. 23 (1922), pp. 292-300,
} 
then

$$
g^{x^{\alpha}}=e^{-h^{2} x^{\infty}}=e^{-x^{2} / 2 \sigma^{2}}
$$

Hence, under the normal probability law, it is asymptotically certain that the greatest variate will be, apart from the factor $\left(1+\varepsilon^{\prime}\right)$,

$$
\left(-\log _{g} n\right)^{1 / a}=\frac{\sqrt{\log _{e} n}}{h}=\sigma \sqrt{2 \log _{e} n} .
$$

These results hold also for a Gram series with but a finite number of terms, since the polynomial factor has no asymptotic influence.

On account of the symmetry of the normal function, an average value for the variation interval is obtained by doubling the corresponding value for the greatest variate. The following table compares the median and asymptotic values of the variation interval, computed by the formulas of this paper, with the modal, mean, and restricted mean ("bedingte ... mathematische Erwartung") values obtained by Bortkiewicz.* Bortkiewicz, indeed, after noting that his mean determination is very close to the average of the other two, gives examples from anthropometry and roulette in which the actual variation is close to his mean value.

Values of $\frac{\text { Variation Interval }}{\sigma}$ for $n$ variates subject to $\frac{1}{\sigma \sqrt{2 \pi}} e^{-x^{2} / 2 \sigma^{2}}$

\begin{tabular}{|r||c|c|c|c|c|c|}
\hline $\begin{array}{c}\text { Number of } \\
\text { variates } \\
n\end{array}$ & $\begin{array}{c}\text { Modal } \\
\text { value } \\
\text { (Bortkiewicz) }\end{array}$ & $\begin{array}{c}\text { Median } \\
\text { value } \\
\text { (Dodd) }\end{array}$ & $\begin{array}{c}\text { Mean } \\
\text { value } \\
\text { (Bortkiewicz) }\end{array}$ & $\begin{array}{c}\text { Restricted } \\
\text { mean value } \\
\text { (Bortkiewicz) }\end{array}$ & $\begin{array}{c}\text { Asymp- } \\
\text { totic } \\
\text { (Dodd) }\end{array}$ & $\begin{array}{c}\text { Asymptotic } \\
\div \\
\text { Median }\end{array}$ \\
\hline 100 & 4.76 & 4.92 & 5.04 & 5.30 & 6.07 & 1.23 \\
1,000 & 6.23 & 6.39 & 6.48 & 6.73 & 7.43 & 1.16 \\
10,000 & 7.47 & 7.62 & 7.70 & 7.92 & 8.58 & 1.13 \\
100,000 & 8.55 & 8.69 & 8.70 & 8.95 & 9.60 & 1.10 \\
\hline
\end{tabular}

The asymptotic value of the interval of variation is thus about $23 \%$ too large when $n=100$, and is still $10 \%$ too large when $n=100,000$.

considers in particular the transformation $x^{\prime \prime}=k \cdot x^{n}$, which would, for example, give the distribution of volumes of similar solids - "oranges" - if the "diameters" are normally distributed. In such a case as this, where $x^{\prime \prime}$ is an increasing function of $x$, the asymptotic value of the greatest "volume" can be found by finding first the asymptotic value of the greatest "diameter".

${ }^{*}$ Loc. cit. See also Nordisk Statistisk Tidskrift, vol. 1, pp. 11-38. 


\section{The Pearson frequency tyPes*}

The Pearson frequency types are given below with the asymptotic interval of variation for each, and the number of the theorem involved.

\section{Asymptotic interval of variation for the Pearson frequency types}

\begin{tabular}{|c|c|c|c|}
\hline $\begin{array}{c}\text { Type } \\
\text { number }\end{array}$ & Frequency function & Interval of variation & $\begin{array}{c}\text { Number } \\
\text { of theorem }\end{array}$ \\
\hline I & $y_{0}\left(1+\frac{x}{\alpha}\right)^{\nu \alpha}\left(1-\frac{x}{\beta}\right)^{\nu \beta}$ & $\alpha+\beta$ & I \\
II & $y_{0}\left(1-\frac{x^{2}}{\alpha^{8}}\right)^{\nu \alpha}$ & $2 \alpha$ & I \\
III & $y_{0}\left(1+\frac{x}{\alpha}\right)^{\nu \alpha} e^{-\nu x}$ & $\alpha+\frac{\log _{e} n}{\nu}$ & I, III \\
IV & $y_{0}\left(1+\frac{x^{8}}{a^{8}}\right)^{-m} e^{-\nu \tan { }^{-1}(x / a)}$ & $2 n^{1 /(2 m-1)}, m>\frac{1}{2}$ & II \\
V & $y_{0} x^{-8} e^{-\gamma / x}, x>0$ & $n^{1 /(8-1)}, s>1$ & I, II \\
VI & $y_{0}(x-a)^{q_{2}} x^{-q_{1}}, x \geqq a$ & $n^{1 /\left(q_{1}-q_{2}-1\right)}-a, q_{1}>q_{8}+1$ & I, II \\
VII & $y_{0} e^{-x^{2} / 2 \sigma^{2}}$ & $2 \sigma \sqrt{2 \log _{e} n}$ & III \\
\hline
\end{tabular}

6. OTHER FREQUENCY FUNCTIONS

1. Jørgensen function. The Jørgensen function is of the formt

$$
k e^{-\frac{1}{2}\left[\frac{\log x-\xi}{\delta}\right]^{2}},
$$

where $k, \xi$, and $\delta$ are constants, and $x>0$. It can be written

where

$$
k^{\prime} x^{\beta^{\prime}} g^{(\log x)^{*}},
$$

$$
\log _{e} g=\frac{-1}{2 \delta^{2}}, \quad \beta^{\prime}=\frac{\xi}{\delta^{2}}, \quad k^{\prime}=\text { constant. }
$$

"Certain special and limiting cases have also been designated as "types".

† See Arne Fisher, loc. cit., p. 241. 
Then, by Theorem IV, the asymptotic value of the greatest variate is

$$
e^{\delta \sqrt{2 \log _{e} n}}\left(1+\varepsilon^{\prime}\right), \text { with }\left|\varepsilon^{\prime}\right|<\varepsilon,
$$

small at pleasure.

2. Poisson exponential function and Charlier $B$-curve. The Poisson function has the form

$$
\frac{e^{-\lambda} \lambda^{x}}{x !}
$$

But, by Stirling's formula,

$$
x !=x^{x} e^{-x} \sqrt{2 \pi x} e^{\theta / 12 x}, \quad 0<\theta<1
$$

For the asymptotic value of the greatest variate, the only significant factor here is $x^{x}$. This asymptotic value, by Theorem VI, is

$$
X\left(1+\varepsilon^{\prime}\right), \text { where } X^{X}=n,\left|\varepsilon^{\prime}\right|<\varepsilon,
$$

small at pleasure. The Charlier $B$-curve for integral variates is obtained from the Poisson function by differencing. But

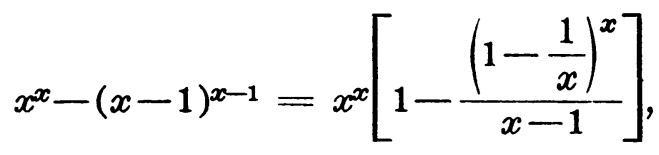

and this bracket has no asymptotic significance. Hence, if only a finite number of terms are taken, the greatest variate, asymptotically, remains that determined as above.

3. Makeham life function. The Makeham formula for the number of survivors at age $x$ from an original group of $l_{0}$ individuals just born is

$$
l_{x}=k s^{x} g^{c^{x}}
$$

where $k>0,0<s<1,0<g<1, c>1$. Postulating a stable population supported by the same number of births annually, and assuming that the theoretic relative frequency is the equivalent of probability, the following table, 
based upon Theorem $\mathrm{V}$, gives the age of the oldest individual, in accordance with constants used in the American Experience Table, as makehamized by Arthur Hunter,* in the Institute of Actuaries Table $\left(\mathrm{H}^{\mathrm{M}}\right)$, and in the McClintock Annuitant Tables, makehamized by W. M. Strong.*

\section{Age of oldest of $n$ individuals}

By asymptotic formula $\log _{c}\left(-\log _{g} n\right)$, where $l_{x}=k s^{x} g^{c^{*}}$.

\begin{tabular}{|c|c|c|c|}
\hline \multirow{3}{*}{ Makehamized mortality table } & \multicolumn{3}{|c|}{ For population of } \\
\hline & one thousand & one million & one billion \\
\hline & oldest age & oldest age & oldest age \\
\hline American experience & 95.1 & 101.7 & 105.5 \\
\hline Institute of Actuaries & 96.3 & 103.9 & 108.3 \\
\hline McClintock-Male ... & 97.8 & 105.3 & 109.7 \\
\hline McClintock-Female. & 100.8 & 108.3 & 112.7 \\
\hline
\end{tabular}

While these results are somewhat crude, it seems surprising that the asymptotic formula which dispenses with the factor $s^{x}$ could do so well. The question, indeed, arises whether any graduation formula can throw much light upon extreme ages, because of the gross irregularities commonly found at the ends of biologic series.

\section{SUMMARY}

The interval of variation is the difference between the greatest and the least of $n$ variates in a distribution. Theorems are here given for the greatest variate; corresponding theorems can be stated for the least variate, using $|x|$ in place of $x$ when necessary. In the following table which summarizes these theorems, the letters stand for positive numbers; they are constants except $x, n$, and $G$. Moreover, $g<1$; but $b>1, c>1, \gamma>1$. For each variate $\int_{x}^{\infty} \varphi(x) d x$ is the probability that the variate will be equal to or greater than $x$. With $\varphi(x)=\varphi_{1}(x) \cdot \psi(x)$, the two factors are each described below. As $n$ increases indefinitely, a probability converges to certainty that the greatest variate will take on the stated asymptotic value, with a relative error small at pleasure for the values in Classes I, III, V, and VI, and for $1 / \alpha$ and $1 / \gamma$ in Classes II and IV.

\footnotetext{
*Transactions of the Actuarial Society of America, vol. 7, p. 200, p. 289.
} 
Asymptotic value of the greatest of $n$ variates

When each variate is subject to $\varphi \cdot(x) \cdot \psi(x){ }^{*}$

\begin{tabular}{|c|c|c|c|c|}
\hline Class & $\varphi_{1}(x)$ & $\begin{array}{l}\text { Conditions } \\
\text { for } \psi(x)\end{array}$ & Greatest variate $\dagger$ & Applications \\
\hline I & $0, x>x_{2}$ & - & $x_{2} \ddagger$ & 5 Pearson Types \\
\hline II & $x^{-1-\alpha}$ & $k_{1}<\psi<k_{2}$ & $n^{1 / a}$ & 3 Pearson Types \\
\hline III & $g^{x^{\alpha}}$ & $x^{-\beta}<\psi<x^{\beta}$ & $\left(-\log _{g} n\right)^{1 / a}$ & $\left\{\begin{array}{l}\text { Gaussian Function } \\
\text { Grams Series (finite) }\end{array}\right.$ \\
\hline IV & $g^{\left(\log _{\mathrm{c}} x\right)^{\gamma}}$ & $x^{-\beta}<\psi<x^{\beta}$ & $c^{\left(-\log _{g} n\right)^{1 / \gamma}}$ & $\left\{\begin{array}{l}\text { Jorgensen Logarithmic } \\
\text { Function }\end{array}\right.$ \\
\hline $\mathrm{V}$ & $g^{c^{x}}$ & $b^{-x}<\psi<b^{x}$ & $\log _{c}\left(-\log _{g} n\right)$ & Makeham Life Function \\
\hline VI & $x^{-x}$ & $b^{-x}<\psi<b^{x}$ & $G$, with $Q^{G}=n$ & $\left\{\begin{array}{l}\text { Poisson Exponential } \\
\text { Charlier } B \text {-Series (finite) }\end{array}\right.$ \\
\hline
\end{tabular}

Asymptotic values have a theoretic importance because of the rigidity of the determination. Possibly, they may be used unreservedly in problems where the variates are as numerous as atoms; but in most practical problems, their chief value would seem to be in furnishing a rough check upon mean, modal, or median values. The latter can be found by determining $G$ so that

$$
\int_{G}^{\infty} \varphi(x) d x=1-2^{-1 / n} .
$$

* Or merely subject when $x$ is sufficiently large.

+ Apart from the factor $\left(1+\varepsilon^{\prime}\right)$, with $\left|\varepsilon^{\prime}\right|<\varepsilon$, small at pleasure.

†Provided $\int_{x_{2}-\varepsilon}^{x_{z}} \varphi_{1}(x) \neq 0$.

UNIVERSITY OF TEXAS, AOSTIN, TeX. 